\title{
Multiscale Approach to Fluid-Solid Interfaces
}

\section{An overview of methodologies coupling fluid mechanics to molecular dynamics and quantum theory}

\author{
Thiago F. Viscondi ${ }^{1}$, Adriano Grigolo ${ }^{1}$, José A. P. Aranha ${ }^{1}$, José R. C. \\ Piqueira $^{1}$, Iberê L. Caldas ${ }^{2}$, and Júlio R. Meneghini ${ }^{1}$ \\ ${ }^{1}$ Escola Politécnica, University of São Paulo, Brazil \\ ${ }^{2}$ Institute of Physics, University of São Paulo, Brazil
}

\begin{abstract}
In conventional fluid mechanics, the chemical composition and thermodynamic state of a fluid-solid interface are not considered when establishing velocity-field boundary conditions. As a consequence, fluid simulations are usually not able to generate different outputs when interfacial materials are varied. By considering an atomistic description of matter, theoretical determination of material-specific boundary conditions becomes possible, thereby providing an improved alternative to the completely-invariant no-slip condition. Such a scheme constitutes a multiscale approach to fluid dynamics involving essentially two transitions between space-time scales: the first concerns the derivation of macroscopic boundary conditions by means of molecular assessment of slip lengths; the second concerns the construction of interatomic force fields, required by molecular dynamics simulations, from quantum theory. In this introductory overview we discuss some of the fundamental aspects of these problems.
\end{abstract}

Keywords: molecular dynamics, boundary conditions, and multiscale methods.

\section{Introduction}

The purpose of fluid mechanics is to predict the motion of a fluid in a given domain when boundary and initial conditions are given. Mathematically, the fluid is regarded as a set of continuum fields - density $\rho$, velocity $\mathbf{u}$, and internal energy $\varepsilon$ - and these must obey balance equations that account for overall mass, momentum, and energy conservation. In their most general form, the balance equations, given below, are written in terms of the stress tensor $\boldsymbol{\sigma}$ and heat flux vector $\mathbf{q}$, which describe, respectively, the exchange of momentum and energy 
between adjacent fluid elements, thus capturing the effects of dissipation.

$$
\begin{aligned}
& \frac{\partial \rho}{\partial t}+\nabla \cdot \rho \mathbf{u}=0, \\
& \frac{\partial \rho \mathbf{u}}{\partial t}+\nabla \cdot(\rho \mathbf{u} \mathbf{u}+\boldsymbol{\sigma})=\rho \mathbf{g}, \\
& \frac{\partial \rho \varepsilon}{\partial t}+\nabla \cdot(\rho \varepsilon \mathbf{u}+\mathbf{q})=-\sum_{i j} \sigma_{i j} \frac{\partial u_{i}}{\partial x_{j}} .
\end{aligned}
$$

In order to close the system of equations, the continuum formulation requires constitutive relations: phenomenological laws that connect $\boldsymbol{\sigma}$ and $\mathbf{q}$ to the basic field variables. Most liquids and gases behave as Newtonian fluids and obey the well-known constitutive laws ${ }^{1}$

$$
\begin{aligned}
& \sigma_{i j}=(p-\eta \nabla \cdot \mathbf{u}) \delta_{i j}-\mu\left(\frac{\partial u_{i}}{\partial x_{j}}+\frac{\partial u_{j}}{\partial x_{i}}-\frac{2}{3} \nabla \cdot \mathbf{u} \delta_{i j}\right), \\
& \mathbf{q}=-\kappa \nabla T .
\end{aligned}
$$

At this level of theory, different substances are therefore characterized by their transport coefficients: shear viscosity $\mu$, volume viscosity $\eta$, and thermal conductivity $\kappa$. Additionally, an equation of state relating pressure $p$, temperature $T$, and density $\rho$ must be supplied, also allowing $\varepsilon$ to be expressed in terms of these quantities.

On the other hand, from a microscopic perspective, the forces experienced by a fluid element, the work done on it, and the amount of heat it absorbs or gives are a result of momentum and energy exchange with the surrounding elements due to molecular diffusion and collisions. Therefore knowledge of the local molecular state of the fluid, in statistical terms, should suffice to specify both $\boldsymbol{\sigma}$ and $\mathbf{q}$ at each point in space. For example, in the case of rarefied gases, the Newtonian constitutive laws emerge from the Boltzmann equation, the fundamental equation of physical kinetics, when non-equilibrium fluctuations are accounted for in the balance equations by means of the Chapman-Enskog and other related procedures. ${ }^{2-4}$ In the case of liquids, formal expressions for the stress tensor and heat current density were derived from statisticalmechanical considerations in a seminal paper by Irving and Kirkwood, ${ }^{5}$ and are routinely used in the microscopic determination of transport coefficients.

In addition to constitutive relations, an appropriate choice of boundary conditions at the fluid-solid interface must be supplied so that the problem expressed in equations (1.1a)-(1.1c) is mathematically well posed. Despite the manifest importance of interfacial boundary conditions, they can not be fully determined within the theoretical framework of macroscopic fluid mechanics. Instead their specification usually relies on empirical observation - in the case of the velocity field of a viscous fluid, the condition at the contact surface between a fluid and a fixed solid wall can be suitably approximated by the no-slip condition, which states, on the grounds of vast experimental evidence, that the flow velocity vanishes at the interface.

Again, from a more fundamental standpoint, the actual condition to be imposed on the flow variables at the fluid-solid interface would be more naturally formulated in terms of the momentum and energy exchange between adjacent fluid and wall elements, being thus determined by the molecular state of the contact region. The information required for specifying this type 
of boundary condition is out of reach of continuum theories - it can only be accessed if the structure of the interface is described at a microscopic level.

It then becomes clear, from the above considerations, that direct microscopic-continuum coupling schemes might be devised where specific constitutive rules and boundary conditions for a given substance are obtained by means of atomistic simulation methods ${ }^{6-10}$ such as molecular dynamics, a term which comprises a set of computer-simulation tools capable of assessing physical properties of materials by performing statistical evaluations on the trajectory of a representative many-molecule system governed by classical equations of motion. ${ }^{11}$

In molecular dynamics simulations, molecules are contained in a finite domain - the simulation box - and interact according to specially designed force fields, the latter usually conveyed through an effective potential energy function. Additionally, the equations of motion can be modified so as to make the system's trajectory sample specific thermodynamical ensembles. ${ }^{12 ; 13}$ Several fluid properties can be reliably obtained from standard equilibrium molecular dynamics: specific heats, equations of state, and even some transport coefficients, such as viscosity and diffusivity; ${ }^{14}$ but there are also ways to enforce non-equilibrium constraints in the simulation box (e.g. strain rate or temperature gradients) and to efficiently measure the material's response - such techniques can be employed, for instance, to determine strain-stress relations in fluids and detect deviations from the Newtonian laws expressed in equations (1.2a) and (1.2b). ${ }^{15 ; 16}$ Similarly, violations of the no-slip condition can be quantified by considering a microscopic setup of the fluid-solid interface where fluid molecules participate in a shear flow.

This type of approach, once complemented with protocols that correctly communicate the information across the micro and macro scales, enables a more detailed fluid-dynamical description, one which is expected to be important not only in the case of unconventional fluids, but also when critical phenomena takes place (e.g. shock waves, phase transitions) in otherwise Newtonian fluids, or when modeling particular regions of the flow such as fluid-fluid and fluid-solid interfaces.

The present contribution provides an overview of the rich physics encountered in such a multiscale framework, taking the boundary-condition specification problem as a guideline. We begin, in section 2, by examining the continuum-molecular link: the key concept of slip length is introduced and the issues related to its evaluation on a fluid-solid interface are discussed, following a brief account of numerical studies on the subject. Then, in section 3, we explore the quantum-mechanical origin of the force fields utilized in molecular simulations, and describe in broad terms the strategies employed for their construction. Final remarks are made in section 4.

\section{Material-specific boundary conditions at fluid-solid in- terfaces}

The investigation of viscous flows past solid surfaces is a central concern of fluid mechanics, as this fundamental issue is ubiquitous in engineering problems. In numerous situations of practical interest the equations governing the flow are solved using the no-slip condition, which asserts that the tangential velocity of the fluid, in the rest frame of the solid surface, is zero

at their interface. ${ }^{17 ; 18}$ Although results obtained under this assumption prove to be consistent 
with most physical observations, one must recognize that the no-slip condition is intrinsically limited, since it essentially states that the shear stress applied by any solid surface on any fluid is always sufficient to bring the flow at the interface to rest. Note that there is no question about the normal component of fluid velocity at the interface, since its value, by the very definition of a flow-constraining solid surface, must be invariably taken as zero. The following discussion then falls only on the tangential velocity of the flow at the fluid-solid interface.

As one might expect, the underlying limitations of the no-slip condition do lead to unphysical or incorrect results in several cases of scientific and technological significance. ${ }^{19-21}$ For example, the no-slip condition causes the divergence of physical quantities, such as the stress tensor and the energy dissipation rate, when trying to describe the contact line of a two-phase fluid moving along a solid surface. ${ }^{22 ; 23}$ The no-slip condition also fails to correctly quantify the velocity fields of internal flows through diminutive channels, such as nanotubes, porous media, membranes, and microfluidic devices. ${ }^{24 ; 25}$ In these systems, surface effects become dominant over the bulk behavior of the fluid, often leading to novel phenomena.

Furthermore, the no-slip condition is material invariant, that is, its prescribed interfacial velocity does not depend on the molecular composition, spatial structure, and thermodynamic conditions of the fluid or the solid surface. For instance, as a consequence of the no-slip condition, the same velocity field would be predicted for the flow of water over hydrophobic and hydrophilic surfaces. The no-slip condition also does not take into consideration whether the fluid is a liquid or gas, whether the solid surface is remarkably rough or perfectly smooth, whether the interface is at an extremely high or particularly low temperature, and so on.

Determination of material-specific boundary conditions can be achieved with the aid of molecular dynamics. In this case, the velocity field at a fluid-solid interface is not preliminarily specified, but obtained as a statistical result arising from the collective behavior of many interacting atoms. That is, by considering a suitable choice of interaction forces between a relatively large number of particles, molecular dynamics simulations are able to accurately determine physical properties of fluids, solids, and their interfaces. Once obtained, materialspecific boundary conditions can guide the development or selection of materials with desired interfacial characteristics for particular applications. For example, an accurate interface modeling could assist in the design of internal coatings capable of reducing the head loss in ducts carrying gas or oil.

\subsection{Slip boundary condition}

All issues associated with the no-slip condition, including its material invariance, can be addressed by introducing the concept of fluid slip, that is, by allowing a mismatch between the tangential velocities of the fluid and the solid surface at their interface. In mathematical terms, this procedure amounts to replacing the no-slip condition, which constitutes a Dirichlet or first-type boundary condition, by the following relation:

$$
u_{t}=L_{s} \frac{\partial u_{t}}{\partial n}
$$

which represents a Robin or third-type boundary condition for the fluid velocity field. In the above equation, $u_{t}$ symbolizes the tangential component of the interfacial fluid velocity, 
considering the solid surface at rest, and $\partial u_{t} / \partial n$ denotes the shear rate at the interface or, equivalently, the derivative of the tangential velocity with respect to the surface normal direction. The parameter $L_{s}$, for which identity (2.1) establishes a definition, is known as slip length.

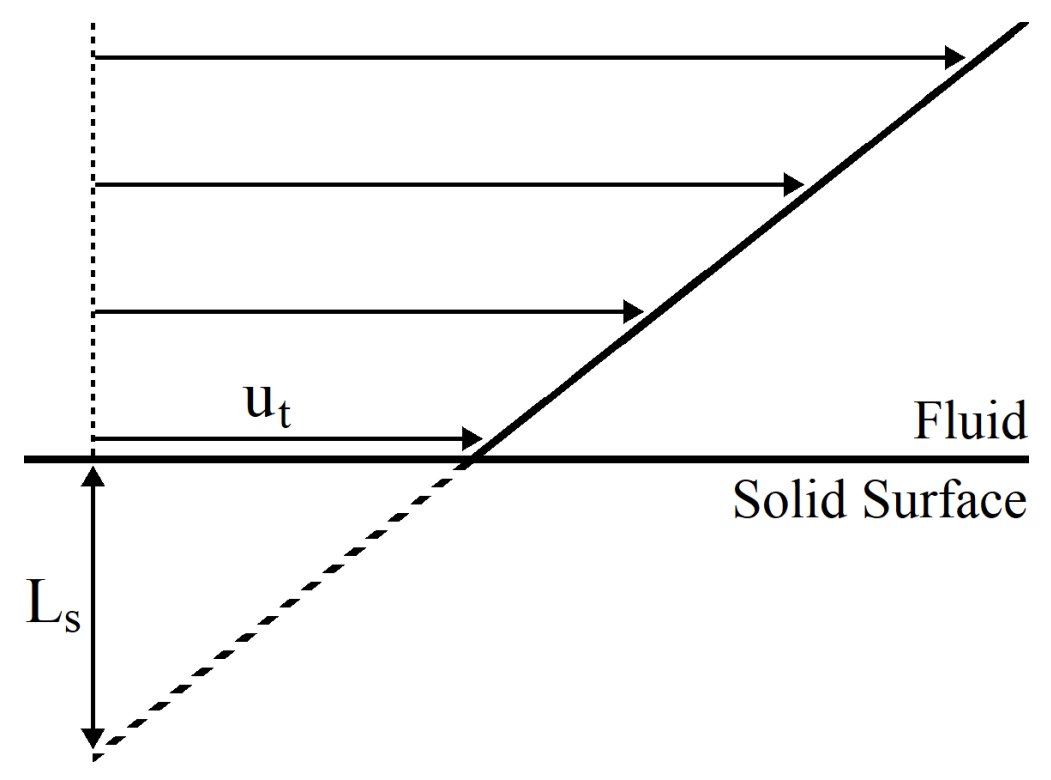

Figure 2.1: Depiction of the slip length $L_{s}$ for a fluid flow at a stationary solid surface.

According to equation (2.1), the slip length can be interpreted as the distance from the interface by which the fluid velocity field needs to be linearly extrapolated in order to achieve the velocity of the solid surface. This geometric interpretation is depicted in figure 2.1.

Note that, by setting $L_{s}=0$ in identity (2.1), the no-slip condition is recovered. On the other hand, when $L_{s}$ goes to infinity, perfect slip is obtained. In general, the slip length establishes a proper quantification for the magnitude of fluid slip over a solid surface. Moreover, the slip-length value can incorporate information about the materials composing the fluid-solid interface, such as roughness and chemical constituents, and also details about the flow itself, such as local shear rate and thermodynamic conditions.

The slip length, for a particular choice of interfacial materials and their thermodynamic states, can be evaluated by a direct application of equation (2.1) to a molecular dynamics simulation of a simple flow configuration, such as a planar Couette ${ }^{\mathrm{i}}$ or a Poiseuille ${ }^{\mathrm{ii}}$ flow, as portrayed by figure 2.2. The procedure of extracting the slip-length value from a molecular dynamics simulation begins by performing local time averages over the particle velocities in a steady flow regime. As a result, the fluid velocity field is obtained, thus making readily available the interfacial values of fluid velocity and shear rate, which are then used to calculate the slip length according to its definition.

\footnotetext{
${ }^{\text {i}}$ Planar Couette flow is the drag-induced flow of a viscous fluid confined between two parallel-moving flat plates.

${ }^{\text {ii }}$ Poiseuille flow is the pressure-induced flow of a viscous fluid confined in a straight duct.
} 


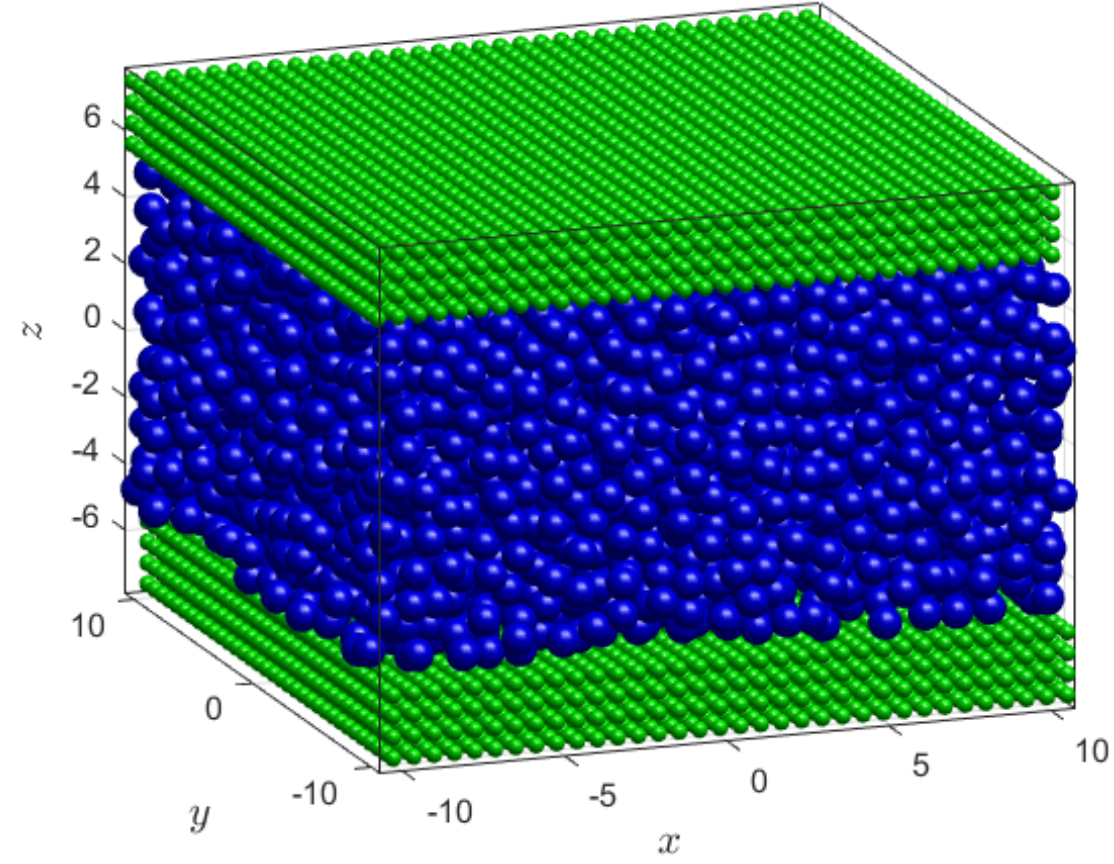

Figure 2.2: Snapshot of a molecular dynamics simulation depicting a monatomic fluid (blue spheres) between two rigid solid walls (green spheres).

\subsection{Practical considerations on the slip length}

Once the slip-length value is determined by an atomistic simulation, identity (2.1) can then be employed in the macroscopic context, this time as a third-type boundary condition. However, three subtle issues must be considered before transitioning from the molecular domain to a continuum description of viscous flows.

First, in order to enable the slip boundary condition to be used in an arbitrary macroscopic problem, regardless of its interface geometry, the slip length must not depend on the flow configuration in which its value is evaluated. That is, for a single choice of interfacial materials and thermodynamic conditions, molecular dynamics simulations of, for example, a Couette or a Poiseuille flow must provide the same slip-length value. This is indeed the case, as shown in published works. ${ }^{26 ; 27}$

Second, in translating an atomistically-evaluated slip length into a macroscopic boundary condition, the behaviour of this physical quantity as a function of the system size must be known, otherwise the results of molecular dynamics can not be expected to remain valid on a completely different spatial scale. In extreme-confinement scenarios, it has been demonstrated that the fluid-slip magnitude can vary substantially with the size of the channel. ${ }^{28-30}$ As previously mentioned, in situations of molecular-size confinement, surface effects may become predominant and, as a consequence, evaluated physical properties may differ considerably from their bulk values. On the other hand, for molecular dynamics simulations of sufficiently large size, the slip-length value becomes insensitive to further increases in the pertinent system dimensions, indicating that the interfacial slip is no longer directly influenced by the central part 
of the fluid flow, which presumably has already reached the bulk regime. In this size-invariant case, the slip length is properly characterized as an interface property ${ }^{\mathrm{iii}}$ and its value can be readily employed on the macroscopic scale. ${ }^{31-33}$

The third concern in using equation (2.1) as a boundary condition is the very fact that the slip length depends on a large number of parameters describing the fluid-solid interface, including physical quantities that correspond to dynamical field variables in the macroscopic context. Therefore, at different interface points and time instants, distinct slip-length values may be supplied to condition (2.1), depending on the local and instantaneous values assumed by the fluid field variables, such as temperature, pressure, and velocity. There are basically two ways of dealing with this issue. First, the slip length could be evaluated in advance as a function of all its parameters, considering a sufficiently wide range of their values. In this case, the required number of molecular dynamics simulations would increase exponentially with the considered number of slip-length parameters. However, this costly procedure would be performed only once for each choice of interfacial materials and its results could be promptly employed in an arbitrary number of macroscopic calculations. As a second option, molecular dynamics simulations could be performed concurrently with macroscopic computations. In this situation, the slip length would be evaluated on the fly, only for the immediately necessary values of its parameters. This method, in comparison with a complete preliminary mapping of the slip-length parametric dependence, would substantially reduce the initial investment of computational resources, while the cost of each multiscale simulation, by itself, would increase.

As consistently reported in the literature, ${ }^{34-36}$ by employing molecular-dynamics simulations, the typical behavior of the slip length as a function of an interface variable can usually be identified. In this case, as a result of a regression analysis, an adjustable curve, generally specified by a small set of parameters, is used to properly describe the functional dependence of the slip length on the examined variable over a fairly wide domain. Once a suitable regression function is found for a particular choice of interfacial materials, the computational cost of repeatedly calculating slip-length values during a multiscale simulation can be substantially reduced, as only a small number of simulations is required to determine the few adjustable parameters characterizing the relation between the slip length and the interface dynamical variable.

\section{$2.3 \quad$ Aspects determining slip length}

Originally, Navier introduced identity (2.1) as a constitutive relation, establishing a linear correspondence between the interfacial values of velocity and shear rate. ${ }^{37}$ For this reason, in the case where slip length is independent of shear rate, equation (2.1) is known as Navier boundary condition. As extensively demonstrated for polymers melts with the aid of molecular dynamics simulations, ${ }^{35 ; 38-45}$ the slip length associated with a non-Newtonian fluid does not remain constant as a function of shear rate. This expected result provides an example of violation of the Navier condition, that is, a situation in which identity (2.1) constitutes a

\footnotetext{
iii That is, a property whose value is determined only by the variables characterizing the structure and dynamics of the fluid-solid interface and, therefore, does not depend on the state of the fluid flow outside the immediate vicinity of the solid surface.
} 
nonlinear relation between velocity and shear rate at the interface.

Surprisingly, molecular dynamics simulations also indicate that slip length is not shearrate invariant even for Newtonian fluids. ${ }^{34 ; 46}$ More precisely, it has been shown that the slip length asymptotically approaches a constant value for decreasing interfacial shear rate, thus approximately satisfying the Navier condition in a low-shear-rate regime. On the other hand, the slip length may also increase indefinitely as the shear rate converges to a critical value. Subsequent work has suggested that slip-length divergence is in fact caused by modelling solid surfaces as completely rigid molecular structures, since similar behaviour had not been observed for flexible walls. ${ }^{47}$ However, further research has shown that a simultaneous rise in the fluid equilibrium temperature, as the shear rate is increased, had been actually responsible for suppressing unbounded values of slip length. ${ }^{36}$ This result illustrates that, in addition to properly describing the interplay between fluid slip and surface stiffness, ${ }^{48 ; 49}$ appropriate choices of energy-dissipation and thermalisation mechanisms, the so-called molecular-dynamics thermostats ${ }^{50}$ are also essential in determining realistic slip-length values, which would adequately represent an empirical situation.

Material-specific boundary conditions are obtained by an accurate representation, from a statistical-physics viewpoint, of the substances composing a fluid-solid interface. In a molecular dynamics simulation, the description of materials is primarily determined by the choice of effective interaction potentials. This basic statement compels the investigation of the slip length through variations of the interatomic force fields. ${ }^{51-54}$ Another important factor affecting the slip-length value is the spatial arrangement of the solid-surface molecules, as discussed in several studies examining the effects of surface patterning and roughness on fluid slip. ${ }^{55-62}$ The third element influencing slip length, as previously implied, is the thermodynamic state of the interface. Both temperature and pressure have a significant impact on the slip-length value, as demonstrated by molecular dynamics simulations. ${ }^{63-65}$ For instance, as shown for simple fluids, ${ }^{36}$ the slip length presents a linear decreasing behaviour for increasing temperature and constant pressure, whereas its value decreases nonlinearly with increasing pressure and constant temperature.

In view of the many different aspects directly involved in evaluating slip length from an atomistic perspective, several efforts have been directed towards understanding the physical mechanisms underlying interfacial slip. ${ }^{66-71}$ Among these studies, special attention has been given to potential correlations between fluid slip and wettability. ${ }^{72-79}$ As expected, molecular dynamics simulations indicate the existence of a quasi-universal relationship, which states that a decrease in wettability is consistently accompanied by an increase in the slip-length value, although exceptions to this rule have also been found. ${ }^{80-83}$

Just as the partial differential equations governing the macroscopic dynamics of viscous fluids must be supplied with interfacial boundary conditions, which need to be evaluated by empirical observations or by an independent theoretical framework acting on a smaller spacetime scale, molecular dynamics simulations must also receive an external input, namely, the classical force fields determining the effective interaction among atoms. The next section examines the intricate problem of obtaining molecular-dynamics force fields from the fundamental theory of matter, quantum mechanics. 


\section{Force fields in molecular dynamics}

Running a molecular dynamics trajectory requires knowledge of the forces experienced by each molecule inside the simulation box. These forces act, by construction, on specific sites of a molecule, most often coinciding with nuclear positions, though other possible locations include bond midpoints or, when dealing with large biomolecules, coarse-grained atomic clusters. Since forces are repeatedly evaluated at every time step of the simulation, computational efficiency demands that they be provided in explicit form. Hence, they are usually derivable from a potential energy $E$, a known function of $M$ 'atomic positions', hereof meaning 'generic force centers', belonging to all molecules of the system, the force on atom $A$ obtained through:

$$
\mathbf{F}_{A}=-\frac{\partial E\left(\mathbf{R}_{1}, \ldots, \mathbf{R}_{M}\right)}{\partial \mathbf{R}_{A}}
$$

Thus, in order to build a sensible force field, one searches for an all-atom energy expression capable of correctly describing the molecular interaction mechanisms most relevant for the substance at hand and which best suits the thermodynamic conditions at play. Despite the existence of many types of force fields, specifically designed for gases, liquids, metals, proteins, polymers, and so on, ${ }^{84-88}$ the pursuit of new, more accurate, more efficient, and more versatile models is a never-ending research topic in computational physics and chemistry. Here, we give a brief account of how force fields can be constructed.

\subsection{Quantum description of a single molecule}

The energy function in equation (3.1) depends on nuclear coordinates only, implying that all information related to the distribution of electronic charge is somehow incorporated into its functional form. To understand how this comes about we start by considering the quantum description of a single molecule, ${ }^{89 ; 90}$ regarded as a collection of $N_{e}$ electrons and $N_{n}$ nuclei interacting through electrostatic forces. ${ }^{\text {iv }}$ The ensuing theory rests on a crucial observation: since nuclei are much heavier than electrons, the time scale for nuclear motion is many times greater than that for electrons. It is then reasonable to assume that electrons immediately adjust to changes in nuclear positions, which allows for electronic and nuclear degrees of freedom to be handled separately in the Schrödinger equation - this is the essential content of the wellknown Born-Oppenheimer approximation. Under this framework, the nuclear coordinates $\{\mathbf{R}\}$ can be independently treated as classical variables that parametrize an effective Hamiltonian (the operator within square brackets in the left-hand side of equation (3.2) below) which acts on electron coordinates $\{\mathbf{r}\}$ only - thus, the molecular problem amounts to finding wave functions $\Psi\left(\mathbf{r}_{1}, \ldots, \mathbf{r}_{N_{e}} ;\{\mathbf{R}\}\right)$ satisfying

$$
\left[-\sum_{i=1}^{N_{e}} \frac{\hbar^{2} \nabla_{i}^{2}}{2 m_{e}}-\sum_{i=1}^{N_{e}} \sum_{A=1}^{N_{n}} \frac{k_{0} Z_{A} e^{2}}{\left|\mathbf{r}_{i}-\mathbf{R}_{A}\right|}+\sum_{i=1}^{N_{e}} \sum_{j>i} \frac{k_{0} e^{2}}{\left|\mathbf{r}_{i}-\mathbf{r}_{j}\right|}+U_{n n}\{\mathbf{R}\}\right] \Psi=E\left(\mathbf{R}_{1}, \ldots, \mathbf{R}_{N_{n}}\right) \Psi
$$

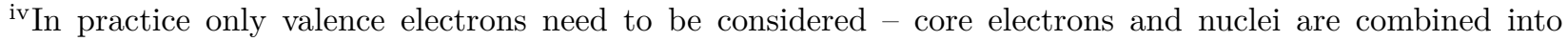
effective charge centers with the help of so-called pseudopotentials.
} 
Here, $\hbar$ is the reduced Planck constant, $m_{e}$ the electron mass, $e$ the elementary charge, $k_{0}$ Coulomb's constant, and $Z_{A}$ is the (effective) atomic number of $A$-th nucleus. The term $U_{n n}\{\mathbf{R}\}$ is the electrostatic repulsion between nuclei and does not depend on electronic coordinates. The energy levels obtained by solving the eigenproblem posed by equation (3.2) are functions of the nuclear positions, varying as the molecular geometry changes - each level constitutes what is known as a potential energy surface, an invaluable concept for understanding many chemical processes (electronic transitions, structural changes, etc.). The surface formed by selecting the lowest energy value at each nuclear configuration is of particular interest, for it describes the normal state of the molecule: its gradients determine the intramolecular forces and its global minimum defines the equilibrium geometry. ${ }^{v}$ Information concerning the molecule's electron charge distribution is built into this energy expression since the latter is an eigenvalue of the electronic wave function.

\subsection{Solving the molecular electronic structure}

Solving the eigenvalue problem (3.2) by standard diagonalisation techniques is not a viable option in a molecular context, except for very small systems, and one must resort to approximate schemes. The most popular and well-established methods employed for this task are the Hartree-Fock approximation ${ }^{91}$ (HF) and density functional theory ${ }^{92 ; 93}$ (DFT), their success owing to their overall balance between accuracy and computational cost. Despite their being founded on different theoretical grounds, both methods are based on the strategy of reducing the many-electron problem to a set of effective single-particle equations that have to be solved self-consistently.

The HF approach is based on the idea that each electron of the system can be approximately described as an independent particle experiencing an average external field due to the presence of the other electrons and nuclei. It outputs optimized single-particle molecular orbitals thus providing an intuitive understanding of electronic processes. The need to evaluate non-local (high-cost) exchange integrals constitutes its major drawback. Meanwhile, DFT rests on the fundamental Hohenberg-Kohn theorems which, in few words, state that the lowest energy of a many-electron system can be obtained through a variational procedure where the electronic density - a very concrete observable (as opposed to the many-body wave function) - is taken as the basic variable. DFT is an exact theory in principle but only approximate in practice because the precise form of the universal energy functional, the central object of the theory, is not known. ${ }^{94}$ It is perhaps not as interpretive as HF but it can be formulated in terms of local (low-cost) integrals only, being more efficient than HF in most cases. It has become routine to compute, using DFT, the electronic structure of molecules containing hundreds of atoms, with overall good agreement between numerical results and experimental measurements. State-of-the-art algorithms are now reaching the thousand-atom regime. ${ }^{95}$

\footnotetext{
${ }^{ }$When more than one potential energy surface is involved in the description, one enters the realm of nonadiabatic dynamics - in photochemistry, for example, laser-induced nuclear vibrations are capable of promoting transitions among otherwise adiabatic energy surfaces.
} 


\subsection{The many-molecule problem and the need for force field tech- niques}

Ideally, one would run an atomistic simulation by solving, at each time step, the electronic structure of the entire system in order to get the required forces: indeed, the formulation so far outlined for isolated molecules can be directly extended to a collection of molecules, in which case the output potential energy surface would be a function of the coordinates of all atoms contained in the simulation box. The envisioned function would then determine, through equation (3.1), both intra- and intermolecular forces at a fixed time instant - the resulting framework would constitute what is called an ab initio molecular-dynamics approach. In the simplest applications, however, one typically has tens of thousands of atoms in the simulation box, and the equations of motion need to be integrated for thousands of time steps. And, while it is still possible to handle the electronic problem for a snapshot of the system, it is simply not affordable to do molecular dynamics in this way, at least not for the sizes (number of atoms) and time spans needed for reliable statistics. Force field methods are precisely designed to overcome this difficulty, and they basically follow two types of strategies, which we may call 'perturbative approach' and 'parameter-fitting approach'.

In a perturbative approach, the energy expression is derived from quantum mechanics by a systematic simplification of the many-molecule problem. ${ }^{96}$ One example of this class of techniques is the Effective Fragment Potential (EFP) method. ${ }^{97-99}$ The essential idea of EFP is to write the interaction energy between molecules, or 'fragments', using a small set of lowcost intermolecular terms together with pre-calculated molecular orbitals from each individual fragment obtained as if they were isolated. The approach is affordable because this last input can be independently computed for each molecule in the system. The method operates at the Hartree-Fock level of theory, where the energy of two nearby fragments depends on a set of parameters that control how the valence electrons of each species redistribute themselves in order to minimize the total energy of the fragment pair. At moderate intermolecular range these parameters are presumably small - one is then able to identify negligible quantities and, following a number of approximations, the mixing parameters can be efficiently computed. In this way, a tractable expression is obtained for the pair's interaction energy, which is later extended to a many-molecule context, although this is not straightforward due to the non-additive character of some of the energy terms (giving rise to so-called many-body effects). ${ }^{100}$ The resulting formulas in EFP are written in terms of quantum ingredients and the various energy contributions - exchange-repulsion, induction, charge transfer, etc. - can be associated with distinct electronic mechanisms. Among other things, this means that EFP can be conveniently employed to interface a molecular dynamics domain (solvent) with a chemically active region (solute), the latter receiving a full quantum treatment - the method was in fact originally intended for this type of application.

In a parameter-fitting approach, on the other hand, the difficulties stemming from the complexity of the quantum problem are circumvented by adopting a completely different strategy. Here, one relies on physical intuition to write the energy as a sum of contributions whose functional dependence on atomic coordinates is designed to model specific types of interactions in an attempt to emulate the behavior that would follow from a complete quantum descrip- 
tion. Usually, bonded (intramolecular) interactions are set to describe simple classical motion - stretching, bending, torsion, vibration, etc. - whereas intermolecular terms involve electrostatic and van der Waals interactions, often including correction factors in order to account for charge-screening and other effects. Each energy term depends on a set of adjustable parameters chosen so that selected features of the substance are correctly reproduced. This optimization can be made with respect to experimental measurements of bulk properties and transport coefficients, or with respect to numerical results from a more sophisticated calculation - for instance, a short-time few-molecule DFT-assisted molecular dynamics simulation. ${ }^{101 ; 102}$ In this latter scheme, a more detailed fitting is possible, since one may resort to experimentally inaccessible information, such as stress components or molecular forces, to carry out the optimization; indeed, it is a popular trend nowadays to employ machine learning algorithms for this task. ${ }^{103}$ The main limitation of this kind of approach is that parameters are necessarily fitted to a predetermined thermodynamic state and thus one should not expect the force field to be accurate under different conditions - the so-called transferability issue. Nevertheless, a well-designed force field can retain its accuracy for a sufficiently broad range of thermodynamic variables and, if this is the case, it will most likely outperform other approaches in practical applications.

\section{$4 \quad$ Final remarks}

By considering the particular problem of determining material-specific boundary conditions at fluid-solid interfaces, two transitions of space-time scale were discussed. First, by introducing the concept of slip length and, thus, establishing a third-type boundary condition, a methodology for the effective application of molecular-dynamics results in the context of macroscopic flows was delineated. In this way, unlike the situation prescribed by the no-slip condition, the dynamics of fluids constrained by solid surfaces acquires a description that depends not only on the chemical composition of the interfacial materials, but also on their thermodynamic states, as specified by field variables such as temperature and pressure, and microscopic spatial configuration, which influences physical characteristics such as surface patterning and roughness. The ability to perform material-specific simulations of macroscopic flows provides a very refined analytical tool, which can assist the design, development, and optimization of technologically relevant devices.

In order to determine the interatomic force fields required for the accurate description of a particular material in a molecular dynamics simulation, a second scale transition was also examined. In this case, brief considerations were made on the employment of numerical methods of quantum mechanics in obtaining effective classical potentials of intra- and intermolecular interaction. In particular, two strategies were addressed: a perturbative approach, which relies on successive simplifications of the complete quantum problem of many interacting particles, and a parametric fitting of potential energy functions, which depends on the comparison with empirical observations or extensive quantum computations.

The multiscale approaches presented in this elementary overview are based on the fact that calculations on different spatial scales can be done independently, that is, physical properties can be evaluated on a finer scale, by considering more fundamental theories of matter, and 
subsequently employed in solving problems on a larger scale, where insufficient theoretical details were initially available. This procedure is only possible because of the great difference also existing on the time scales characterizing distinct physical phenomena, so that the dynamics of a system and its small-sized subsystems can be treated as effectively decoupled.

\section{Acknowledgments}

This work is part of a project developed in the Research Centre for Gas Innovation (RCGI), with support from Shell and FAPESP (Fundação de Amparo à Pesquisa do Estado de São Paulo), under process numbers 2014/50279-4 and 2018/03211-6.

\section{References}

[1] L. D. Landau and E. M. Lifshitz. Fluid mechanics. Pergamon Press, 1987.

[2] Harold Grad. On the kinetic theory of rarefied gases. Communications on Pure and Applied Mathematics, 2(4):331-407, 1949.

[3] Sydney Chapman, Thomas George Cowling, and David Burnett. The mathematical theory of non-uniform gases: an account of the kinetic theory of viscosity, thermal conduction and diffusion in gases. Cambridge university press, 1990.

[4] Gilberto Medeiros Kremer. Uma Introdução à Equação de Boltzmann. Edusp, 2005.

[5] J. H. Irving and John G. Kirkwood. The statistical mechanical theory of transport processes. IV. the equations of hydrodynamics. The Journal of Chemical Physics, 18(6):817$829,1950$.

[6] Nikolaos Asproulis, Marco Kalweit, and Dimitris Drikakis. A hybrid molecular continuum method using point wise coupling. Advances in Engineering Software, 46(1):85-92, 2012.

[7] Barry Z. Shang, Nikolaos K. Voulgarakis, and Jhih-Wei Chu. Fluctuating hydrodynamics for multiscale modeling and simulation: Energy and heat transfer in molecular fluids. The Journal of Chemical Physics, 137(4):044117, jul 2012.

[8] Matthew K. Borg, Duncan A. Lockerby, and Jason M. Reese. A hybrid molecularcontinuum simulation method for incompressible flows in micro/nanofluidic networks. Microfluidics and Nanofluidics, 15(4):541-557, 2013.

[9] Ian A. Cosden and Jennifer R. Lukes. A hybrid atomistic-continuum model for fluid flow using LAMMPS and OpenFOAM. Computer Physics Communications, 184(8):19581965, 2013. 
[10] Anton Markesteijn, Sergey Karabasov, Arturs Scukins, Dmitry Nerukh, Vyacheslav Glotov, and Vasily Goloviznin. Concurrent multiscale modelling of atomistic and hydrodynamic processes in liquids. Philosophical Transactions of the Royal Society A: Mathematical, Physical and Engineering Sciences, 372(2021):20130379-20130379, jun 2014.

[11] Michael P. Allen and Dominic J. Tildesley. Computer simulation of liquids. Oxford university press, 2017.

[12] William G. Hoover. Canonical dynamics: equilibrium phase-space distributions. Physical review $A, 31(3): 1695,1985$.

[13] William G. Hoover. Constant-pressure equations of motion. Physical Review A, 34(3):2499, 1986.

[14] Dennis C. Rapaport. The Art of Molecular Dynamics Simulation. Cambridge university press, 2004.

[15] Roger Edberg, G. P. Morriss, and Denis J. Evans. Rheology of n-alkanes by nonequilibrium molecular dynamics. The Journal of Chemical Physics, 86(8):4555-4570, 1987.

[16] Gary P. Morriss and Denis J. Evans. Statistical Mechanics of Nonequilbrium Liquids. ANU Press, 2007.

[17] Michael A. Day. The no-slip condition of fluid dynamics. Erkenntnis, 33(3):285-296, 1990.

[18] Eric Lauga, Michael Brenner, and Howard Stone. Microfluidics: the no-slip boundary condition. In Springer Handbook of Experimental Fluid Mechanics, pages 1219-1240. Springer, 2007.

[19] Steve Granick, Yingxi Zhu, and Hyunjung Lee. Slippery questions about complex fluids flowing past solids. Nature Materials, 2(4):221, 2003.

[20] Jonathan S. Ellis and Michael Thompson. Slip and coupling phenomena at the liquidsolid interface. Physical Chemistry Chemical Physics, 6(21):4928-4938, 2004.

[21] Chiara Neto, Drew R. Evans, Elmar Bonaccurso, Hans-Jürgen Butt, and Vincent S. J. Craig. Boundary slip in newtonian liquids: a review of experimental studies. Reports on Progress in Physics, 68(12):2859, 2005.

[22] Peter A. Thompson and Mark O. Robbins. Simulations of contact-line motion: slip and the dynamic contact angle. Physical Review Letters, 63(7):766, 1989.

[23] Joel Koplik and Jayanth R. Banavar. No-slip condition for a mixture of two liquids. Physical Review Letters, 80(23):5125, 1998. 
[24] V. P. Sokhan, D. Nicholson, and N. Quirke. Fluid flow in nanopores: an examination of hydrodynamic boundary conditions. The Journal of Chemical Physics, 115(8):3878-3887, 2001.

[25] Vladimir P. Sokhan, David Nicholson, and Nicholas Quirke. Fluid flow in nanopores: Accurate boundary conditions for carbon nanotubes. The Journal of Chemical Physics, 117(18):8531-8539, 2002.

[26] Joel Koplik, Jayanth R. Banavar, and Jorge F. Willemsen. Molecular dynamics of fluid flow at solid surfaces. Physics of Fluids A: Fluid Dynamics, 1(5):781-794, 1989.

[27] Marek Cieplak, Joel Koplik, and Jayanth R. Banavar. Boundary conditions at a fluid-solid interface. Physical Review Letters, 86(5):803, 2001.

[28] S. A. Gupta, H. D. Cochran, and P. T. Cummings. Shear behavior of squalane and tetracosane under extreme confinement. i. model, simulation method, and interfacial slip. The Journal of Chemical Physics, 107(23):10316-10326, 1997.

[29] A. Jabbarzadeh, J. D.Atkinson, and R. I. Tanner. Wall slip in the molecular dynamics simulation of thin films of hexadecane. The Journal of Chemical Physics, 110(5):26122620, 1999.

[30] Chong Liu and Zhigang Li. On the validity of the navier-stokes equations for nanoscale liquid flows: The role of channel size. AIP Advances, 1(3):032108, 2011.

[31] Jinliang $\mathrm{Xu}$ and Yuxiu Li. Boundary conditions at the solid-liquid surface over the multiscale channel size from nanometer to micron. International Journal of Heat and Mass Transfer, 50(13-14):2571-2581, 2007.

[32] John A. Thomas and Alan J. H. McGaughey. Reassessing fast water transport through carbon nanotubes. Nano Letters, 8(9):2788-2793, 2008.

[33] Bladimir Ramos-Alvarado, Satish Kumar, and G. P. Peterson. Hydrodynamic slip length as a surface property. Physical Review E, 93(2):023101, 2016.

[34] Peter A. Thompson and Sandra M. Troian. A general boundary condition for liquid flow at solid surfaces. Nature, 389(6649):360, 1997.

[35] Nikolai V. Priezjev and Sandra M. Troian. Molecular origin and dynamic behavior of slip in sheared polymer films. Physical Review Letters, 92(1):018302, 2004.

[36] Amir Alizadeh Pahlavan and Jonathan B. Freund. Effect of solid properties on slip at a fluid-solid interface. Physical Review E, 83(2):021602, 2011.

[37] C. L. M. H. Navier. Mémoire sur les lois du mouvement des fluides. Mémoires de l'Académie Royale des Sciences de l'Institut de France, 6(1823):389-440, 1823. 
[38] Rajesh Khare, Juan J. de Pablo, and Arun Yethiraj. Rheology of confined polymer melts. Macromolecules, 29(24):7910-7918, 1996.

[39] A. Jabbarzadeh, J. D. Atkinson, and R. I. Tanner. The effect of branching on slip and rheological properties of lubricants in molecular dynamics simulation of couette shear flow. Tribology International, 35(1):35-46, 2002.

[40] Anoosheh Niavarani and Nikolai V. Priezjev. Rheological study of polymer flow past rough surfaces with slip boundary conditions. The Journal of Chemical Physics, 129(14):144902, 2008.

[41] Anoosheh Niavarani and Nikolai V. Priezjev. Slip boundary conditions for shear flow of polymer melts past atomically flat surfaces. Physical Review E, 77(4):041606, 2008.

[42] Nikolai V. Priezjev. Shear rate threshold for the boundary slip in dense polymer films. Physical Review E, 80(3):031608, 2009.

[43] Nikolai V. Priezjev. Relationship between induced fluid structure and boundary slip in nanoscale polymer films. Physical Review E, 82(5):051603, 2010.

[44] Nikolai V. Priezjev. Interfacial friction between semiflexible polymers and crystalline surfaces. The Journal of chemical physics, 136(22):224702, 2012.

[45] Nikolai V. Priezjev. Fluid structure and boundary slippage in nanoscale liquid films. Detection of Pathogens in Water Using Micro and Nano-technology, 2012.

[46] Nikolai V. Priezjev. Rate-dependent slip boundary conditions for simple fluids. Physical Review E, 75(5):051605, 2007.

[47] Ashlie Martini, Hua-Yi Hsu, Neelesh A. Patankar, and Seth Lichter. Slip at high shear rates. Physical Review Letters, 100(20):206001, 2008.

[48] Nikolaos Asproulis and Dimitris Drikakis. Boundary slip dependency on surface stiffness. Physical Review E, 81(6):061503, 2010.

[49] Nikolaos Asproulis and Dimitris Drikakis. Wall-mass effects on hydrodynamic boundary slip. Physical Review E, 84(3):031504, 2011.

[50] Philippe H. Hünenberger. Thermostat algorithms for molecular dynamics simulations. In Advanced Computer Simulation, pages 105-149. Springer, 2005.

[51] Chong Liu and Zhigang Li. Flow regimes and parameter dependence in nanochannel flows. Physical Review E, 80(3):036302, 2009.

[52] Chong Liu and Zhigang Li. Surface effects on nanoscale poiseuille flows under large driving force. The Journal of Chemical Physics, 132(2):024507, 2010. 
[53] D. Toghraie Semiromi and A. R. Azimian. Nanoscale poiseuille flow and effects of modified lennard-jones potential function. Heat and Mass Transfer, 46(7):791-801, 2010.

[54] Filippos Sofos, Theodoros E. Karakasidis, and Antonios Liakopoulos. Parameters affecting slip length at the nanoscale. Journal of Computational and Theoretical Nanoscience, 10(3):648-650, 2013.

[55] A. Jabbarzadeh, J. D. Atkinson, and R. I. Tanner. Effect of the wall roughness on slip and rheological properties of hexadecane in molecular dynamics simulation of couette shear flow between two sinusoidal walls. Physical Review E, 61(1):690, 2000.

[56] Cécile Cottin-Bizonne, Jean-Louis Barrat, Lydéric Bocquet, and Elisabeth Charlaix. Lowfriction flows of liquid at nanopatterned interfaces. Nature Materials, 2(4):237, 2003.

[57] Cécile Cottin-Bizonne, Catherine Barentin, Élisabeth Charlaix, Lydéric Bocquet, and J.-L. Barrat. Dynamics of simple liquids at heterogeneous surfaces: Molecular-dynamics simulations and hydrodynamic description. The European Physical Journal E, 15(4):427438, 2004.

[58] Toni-Marie Galea and Phil Attard. Molecular dynamics study of the effect of atomic roughness on the slip length at the fluid- solid boundary during shear flow. Langmuir, 20(8):3477-3482, 2004.

[59] Nikolai V. Priezjev, Anton A. Darhuber, and Sandra M. Troian. Slip behavior in liquid films on surfaces of patterned wettability: Comparison between continuum and molecular dynamics simulations. Physical Review E, 71(4):041608, 2005.

[60] Nikolai V. Priezjev and Sandra M. Troian. Influence of periodic wall roughness on the slip behaviour at liquid/solid interfaces: molecular-scale simulations versus continuum predictions. Journal of Fluid Mechanics, 554:25-46, 2006.

[61] Nikolai V. Priezjev. Effect of surface roughness on rate-dependent slip in simple fluids. The Journal of Chemical Physics, 127(14):144708, 2007.

[62] Anoosheh Niavarani and Nikolai V. Priezjev. Modeling the combined effect of surface roughness and shear rate on slip flow of simple fluids. Physical Review E, 81(1):011606, 2010.

[63] Zhaoli Guo, T. S. Zhao, and Yong Shi. Temperature dependence of the velocity boundary condition for nanoscale fluid flows. Physical Review E, 72(3):036301, 2005.

[64] J. Servantie and M. Müller. Temperature dependence of the slip length in polymer melts at attractive surfaces. Physical Review Letters, 101(2):026101, 2008.

[65] Luyao Bao, Nikolai V. Priezjev, Haibao Hu, and Kai Luo. Effects of viscous heating and wall-fluid interaction energy on rate-dependent slip behavior of simple fluids. Physical Review E, 96(3):033110, 2017. 
[66] Seth Lichter, Alex Roxin, and Shreyas Mandre. Mechanisms for liquid slip at solid surfaces. Physical Review Letters, 93(8):086001, 2004.

[67] Seth Lichter, Ashlie Martini, Randall Q. Snurr, and Qian Wang. Liquid slip in nanoscale channels as a rate process. Physical Review Letters, 98(22):226001, 2007.

[68] A. Martini, A. Roxin, R. Q. Snurr, Q. Wang, and S. Lichter. Molecular mechanisms of liquid slip. Journal of Fluid Mechanics, 600:257-269, 2008.

[69] Xin Yong and Lucy T. Zhang. Investigating liquid-solid interfacial phenomena in a couette flow at nanoscale. Physical Review E, 82(5):056313, 2010.

[70] Taha Sochi. Slip at fluid-solid interface. Polymer Reviews, 51(4):309-340, 2011.

[71] Xin Yong and Lucy T. Zhang. Slip in nanoscale shear flow: mechanisms of interfacial friction. Microfluidics and Nanofluidics, 14(1-2):299-308, 2013.

[72] Jean-Louis Barrat and Lydéric Bocquet. Large slip effect at a nonwetting fluid-solid interface. Physical Review Letters, 82(23):4671, 1999.

[73] Jean-Louis Barrat and Lydéric Bocquet. Influence of wetting properties on hydrodynamic boundary conditions at a fluid/solid interface. Faraday Discussions, 112:119-128, 1999.

[74] Gyoko Nagayama and Ping Cheng. Effects of interface wettability on microscale flow by molecular dynamics simulation. International Journal of Heat and Mass Transfer, 47(3):501-513, 2004.

[75] David M. Huang, Christian Sendner, Dominik Horinek, Roland R. Netz, and Lydéric Bocquet. Water slippage versus contact angle: A quasiuniversal relationship. Physical Review Letters, 101(22):226101, 2008.

[76] Christian Sendner, Dominik Horinek, Lyderic Bocquet, and Roland R. Netz. Interfacial water at hydrophobic and hydrophilic surfaces: Slip, viscosity, and diffusion. Langmuir, 25(18):10768-10781, 2009.

[77] Kai Huang and Izabela Szlufarska. Friction and slip at the solid/liquid interface in vibrational systems. Langmuir, 28(50):17302-17312, 2012.

[78] Bladimir Ramos-Alvarado, Satish Kumar, and G. P. Peterson. Wettability transparency and the quasiuniversal relationship between hydrodynamic slip and contact angle. Applied Physics Letters, 108(7):074105, 2016.

[79] Tsu-Hsu Yen and Chyi-Yeou Soong. Effective boundary slip and wetting characteristics of water on substrates with effects of surface morphology. Molecular Physics, 114(6):797809, 2016. 
[80] Roman S. Voronov, Dimitrios V. Papavassiliou, and Lloyd L. Lee. Boundary slip and wetting properties of interfaces: Correlation of the contact angle with the slip length. The Journal of Chemical Physics, 124(20):204701, 2006.

[81] Roman S. Voronov, Dimitrios V. Papavassiliou, and Lloyd L. Lee. Slip length and contact angle over hydrophobic surfaces. Chemical Physics Letters, 441(4-6):273-276, 2007.

[82] Roman S. Voronov, Dimitrios V. Papavassiliou, and Lloyd L. Lee. Review of fluid slip over superhydrophobic surfaces and its dependence on the contact angle. Industrial $\mathscr{6}$ Engineering Chemistry Research, 47(8):2455-2477, 2008.

[83] Tuan Anh Ho, Dimitrios V. Papavassiliou, Lloyd L. Lee, and Alberto Striolo. Liquid water can slip on a hydrophilic surface. Proceedings of the National Academy of Sciences, 2011.

[84] Bernard R. Brooks, Robert E. Bruccoleri, Barry D. Olafson, David J. States, S. Swaminathan, and Martin Karplus. Charmm: a program for macromolecular energy, minimization, and dynamics calculations. Journal of Computational Chemistry, 4(2):187-217, 1983.

[85] Murray S. Daw and Michael I. Baskes. Embedded-atom method: Derivation and application to impurities, surfaces, and other defects in metals. Physical Review B, 29(12):6443, 1984.

[86] Adri C. T. van Duin, Siddharth Dasgupta, Francois Lorant, and William A. Goddard. Reaxff: a reactive force field for hydrocarbons. The Journal of Physical Chemistry A, 105(41):9396-9409, 2001.

[87] Jay W. Ponder and David A. Case. Force fields for protein simulations. In Advances in Protein Chemistry, volume 66, pages 27-85. Elsevier, 2003.

[88] Pedro E. M. Lopes, Olgun Guvench, and Alexander D. MacKerell. Current status of protein force fields for molecular dynamics simulations. In Molecular Modeling of Proteins, pages 47-71. Springer, 2015.

[89] Peter W. Atkins and Ronald S. Friedman. Molecular quantum mechanics. Oxford university press, 2011.

[90] Attila Szabo and Neil S. Ostlund. Modern quantum chemistry: introduction to advanced electronic structure theory. Courier Corporation, 2012.

[91] John Clarke Slater. Quantum theory of molecules and solids, volume 1. McGraw-Hill New York, 1963.

[92] Pierre Hohenberg and Walter Kohn. Inhomogeneous electron gas. Physical Review, 136(3B):B864, 1964. 
[93] Walter Kohn and Lu Jeu Sham. Self-consistent equations including exchange and correlation effects. Physical review, 140(4A):A1133, 1965.

[94] Klaus Capelle. A bird's-eye view of density-functional theory. Brazilian Journal of Physics, 36(4A):1318-1343, 2006.

[95] Laura E. Ratcliff, Stephan Mohr, Georg Huhs, Thierry Deutsch, Michel Masella, and Luigi Genovese. Challenges in large scale quantum mechanical calculations. Wiley Interdisciplinary Reviews: Computational Molecular Science, 7(1):e1290, 2017.

[96] Peng Xu, Emilie B. Guidez, Colleen Bertoni, and Mark S. Gordon. Perspective: Ab initio force field methods derived from quantum mechanics. Journal of Chemical Physics, 148(9):090901, 2018.

[97] Mark S. Gordon, Mark A. Freitag, Pradipta Bandyopadhyay, Jan H. Jensen, Visvaldas Kairys, and Walter J. Stevens. The Effective Fragment Potential Method: A QM-Based MM Approach to Modeling Environmental Effects in Chemistry. The Journal of Physical Chemistry A, 105(2):293-307, 2001.

[98] Jan H. Jensen. Intermolecular exchange-induction and charge transfer: Derivation of approximate formulas using nonorthogonal localized molecular orbitals. The Journal of Chemical Physics, 114(20):8775-8783, may 2001.

[99] Mark S. Gordon, Quentin A. Smith, Peng Xu, and Lyudmila V. Slipchenko. Accurate First Principles Model Potentials for Intermolecular Interactions. Annual Review of Physical Chemistry, 64(1):553-578, 2013.

[100] Anthony Stone. The theory of intermolecular forces. OUP Oxford, 2013.

[101] P. Tangney and S. Scandolo. An ab initio parametrized interatomic force field for silica. The Journal of Chemical Physics, 117(19):8898-8904, nov 2002.

[102] R. Tangney and S. Scandolo. A many-body interatomic potential for ionic systems: Application to MgO. Journal of Chemical Physics, 119(18):9673-9685, 2003.

[103] Matthias Rupp, O. Anatole von Lilienfeld, and Kieron Burke. Guest editorial: Special topic on data-enabled theoretical chemistry, 2018. 